\title{
ASYMMETRIC MHD STELLAR WINDS AND RELATED FLOWS
}

\author{
K. TSINGANOS \\ Department of Physics, University of Crete and \\ Research Center of Crete, GR - 71409 \\ Heraklion, Crete, Greece
}

Some well established observational facts about most wind-type astrophysical outflows are the following: (i) they are strongly nonspherically symmetric and at least two-dimensional; fast solar wind streams from polar coronal holes and jets from stars and galaxies are two representative and characteristic examples. (ii) some kind of nonthermal heating is required, at least during the initial acceleration stage of the outflow; therefore, the flow is far from beeing adiabatic and the assumption of polytropicity with an arbitrarily specified index $\gamma$, although useful from the mathematical point of view to solve the governing equations and provide some physical insight into the problem, is nevertheless artificial. (iii) the ubiquitous magnetic field seems to play a decisive direct, or at least, indirect role in heating stellar coronae and driving stellar winds. Nevertheless - perhaps for the sake of simplicity - most studies on astrophysical outflows and winds so far have neglected to incorporate the above three basic features of nonspherical expansion, nonpolytropic equation of state and magnetohydrodynamic description of the problem. We have recently embarked in an effort to model wind-type outflows by incorporating those basic physical constraints (Low and Tsinganos, 1986; Tsinganos and Low, 1989; Tsinganos and Trussoni, 1990, 1991; Tsinganos and Sauty, 1992a,b).

The starting point of our studies is the full set of the MHD equations,

$$
\begin{gathered}
\nabla \cdot \vec{B}=\nabla \cdot(\rho \vec{V})=\nabla \times(\vec{V} \times \vec{B})=0, \\
\rho(\vec{V} \cdot \nabla) \vec{V}=-\nabla P+\frac{1}{4 \pi}(\nabla \times \vec{B}) \times \vec{B}-\frac{\rho G M}{r^{2}} \vec{e}_{r}, \\
3\left(\frac{k}{m_{p}}\right) \rho(\vec{V} \cdot \nabla) T-2\left(\frac{k}{m_{p}}\right) \rho(\vec{V} \cdot \nabla) \rho=\rho \sigma, T=\frac{m_{p}}{2 k} \frac{P}{\rho},
\end{gathered}
$$

where the symbols have their usual meaning. The system of Eqs. (2.1) is closed with the conservation of energy law taken directly from the first law of thermodynamics where $\rho \sigma(R, \theta)$ is the rate of some energy deposition per unit volume of the fluid. The resulting value of the effective variable polytropic index along each streamline, $\gamma \equiv \operatorname{dln} P / \operatorname{dln} \rho \neq$ const. may then be compared to some characteristic values, such as $\gamma=1$ (isothermal atmosphere), $\gamma=3 / 2$ (Parker polytrope), or $\gamma=5 / 3$ (adiabatic expansion).

In spherical coordinates $(R, \theta, \phi)$ with $R$ the dimensionless radial distance and $\theta$ the colatitude, the following hydromagnetic field,

$$
V_{r}(R, \theta)=V_{o} Y(R) f(R) \frac{\cos \theta}{\left[1+\omega f \sin ^{2} \theta\right]^{1 / 2}} \quad, \quad B_{r}(R, \theta)=\frac{B_{\circ} f}{R^{2}} \cos \theta,
$$




$$
\begin{gathered}
V_{\theta}(R, \theta)=-V_{o} \frac{Y R}{2} \frac{d f}{d R} \frac{\sin \theta}{\sqrt{\left(1+\omega f \sin ^{2} \theta\right)}}, B_{\theta}(R, \theta)=-\frac{B_{o}}{2 R} \frac{d f}{d R} \sin \theta, \quad(2.2 \mathrm{~b}) \\
V_{\varphi}(R, \theta)=\lambda V_{o} R \frac{\sin \theta}{\sqrt{\left(1+\omega f \sin ^{2} \theta\right)}}\left(\frac{Y f-Y_{\star} f_{\star}}{1-M_{a}^{2}}\right), B_{\varphi}(R, \theta)=\frac{\lambda B_{o}}{R Y} \sin \theta\left(\frac{f Y-M_{a}^{2} f_{\star} Y_{\star}}{1-M_{a}^{2}}\right),
\end{gathered}
$$

with $V_{o}^{A}=B_{o} /\left(4 \pi \rho_{o}\right)^{1 / 2}, \beta=V_{o}^{A} / V_{o}, M_{A}^{2}=\left[V_{r} / V_{A}\right]^{2}=4 \pi \rho V_{r}^{2} / B_{r}^{2}=Y R^{2} / \beta^{2}, R_{*}$ the Alfvenic transition where $M_{A}=1$ and $f_{\star}=f\left(R_{\star}\right), Y_{\star}=Y\left(R_{\star}\right)$ satisfy the conservation laws (2.1a,b), as well as the $\phi$-component of the momentum balance equation, if the density and pressure are given by the expressions

$$
\rho(R, \theta)=\frac{\rho_{o}}{Y(R) R^{2}}\left[1+\omega f \sin ^{2} \theta\right], P(R, \theta)=P_{o}(R)+P_{1}(R) \sin ^{2} \theta .
$$

where $\rho_{o}$ is the density at the polar base $(R=1, \theta=0)$ and $\omega, \lambda$ are constants.

The remaining $r$ - and $\theta$-components of the force balance equation yield then three ODE's for $f(R), Y(R), P_{o}(R)$ and $P_{1}(R)$. By specifying $f(R)$, or setting $P_{1}(R)=\kappa P_{o}(R)$, we may solve for the other three unknowns. However, this system of the three coupled ordinary differential equations has several novel critical points. For example, if $f(R)=1$, (Tsinganos and Trussoni 1991) we have a first order X-type singularity together with a higher order sink-type Alfvenic singularity which together select a unique characteristic wind-type solution. On the other hand, if $P_{1}(R)=\kappa P_{o}(R)$ we obtain several nodal and saddle critical points that again select a unique wind type solution (Tsinganos and Sauty $1992 b)$.

Note that several parameters denote the deviations of the ontflow from the classical Parker solution. Consider first, $\omega$ that denotes the degree of latitudinal density inhomogeneity and velocity collimation. Large values of $\omega$-meaning polar density depletion relative to the lower latitudes and also high velocity collimation - yield high polar acceleration and larger polar terminal speeds, as in solar coronal holes. Second, the more magnetized is the outflow (large values of the magnetic parameter $\beta$ ) the larger is the asymptotic speed and the heating close to the base is more isothermal-like extending over larger distances. Thus, a flat temperature distribution in the framework of this model is an indication of a magnetically dominated outflow. Third, the lower is the polar pressure relative to the equatorial one, and the higher is the amplitude of rotation, $\lambda$, the lower is the initial acceleration and terminal speed. The reason is that in this model, where the radial flow speed is zero at the equator, there the plasma weight is larger than the pressure gradient by the magnitude of the centrifugal force, or the equatorial pressure en hancement. At the pole then, where there is no such centrifugal force, or the pressure is less, the plasma weight takes over the pressure gradient and the acceleration reduces. Fourth, the character of the acceleration and terminal speed is very sensitive to the initial degree of flaring of the streamlines. In fact, streamline flaring toward the equator reduces the acceleration contrary to what happens in polytropic flows - and only poleward flaring enhances the terminal speed. Finally, the effective polytropic index $\gamma$ which becomes asymptotically 1 is an indication of the widespread heating required in magnetically dominated winds.

\section{References}

Low, B.C. and Tsinganos, K.: 1986, Astrophys. J. 302, 163.

Tsinganos, K. and Low, B.C.: 1989, Astrophys. J. 342, 1028.

Tsinganos, K. and Trussoni, E.: 1990, Astron. Astrophys. 231, 270.

Tsinganos, K. and Trussoni, E.: 1991, Astron. Astrophys., (in press).

Tsinganos, K. and Sauty, C.: 1992a,b Astron. Astrophys., (in press). 